\title{
A associação entre a suspeita inicial de perda auditiva e a ausência de comunicação verbal em crianças com transtornos do espectro autístico
}

\author{
The association between an initial suspicion of hearing loss \\ and the absence of verbal communication in children with \\ autism spectrum disorders
}

\author{
Elaine Colombo Sousa ${ }^{1}$, Fernanda Thieme Lima ${ }^{2}$, Ana Carina Tamanaha ${ }^{3}$, Jacy Perissinoto ${ }^{4}$, \\ Marisa Frasson de Azevedo ${ }^{5}$, Brasília Maria Chiari ${ }^{6}$
}

\begin{abstract}
RESUMO
Objetivo: Averiguar a associação entre a suspeita inicial de deficiência auditiva e a ausência de comunicação verbal em crianças com diagnóstico de transtornos do espectro autístico. Métodos: Por meio de estudo retrospectivo, analisamos 54 anamneses de indivíduos com diagnóstico de transtornos do espectro autístico. Inicialmente, obtivemos o registro da suspeita inicial de perda auditiva, mencionada pela família. O grau de associação entre a presença de suspeita inicial de perda auditiva e o tipo de desempenho comunicativo (não verbal e verbal) foi verificado. Posteriormente, as avaliações audiológicas foram rastreadas para confirmarmos a pertinência da queixa auditiva mencionada. Para tratamento estatístico dos resultados utilizamos os Testes Qui-quadrado e Exato de Fisher. Estabeleceu-se nível de significância de 0,05. Resultados: Verificamos que 62,96\% dos pais de crianças autistas (verbais ou não) apresentaram a suspeita inicial de perda auditiva como primeira etiologia. A avaliação audiológica mostrou que somente $11,11 \%$ dos indivíduos com comunicação não verbal e nenhum com comunicação verbal apresentaram algum tipo de deficiência auditiva. Conclusões: $\mathrm{O}$ número de crianças com suspeita inicial de perda auditiva mostrou-se significativo, independente destas serem verbais ou não. Na análise da associação entre desempenho comunicativo e suspeita de prejuízos na audição, os resultados foram estatisticamente significante maiores em crianças não verbais. Esses dados nos fazem refletir sobre a importância da sensibilização dos profissionais que atuam em clínica infantil quanto à busca pelo diagnóstico diferencial entre a deficiência auditiva e os transtornos do espectro autístico.
\end{abstract}

Descritores: Perda auditiva/complicações; Transtorno autístico; Linguagem infantil; Comportamento verbal; Transtornos da linguagem

Trabalho realizado no Núcleo de Investigação Fonoaudiológica da Linguagem nos Transtornos Globais do Desenvolvimento (NIFTGD) do Departamento de Fonoaudiologia da Universidade Federal de São Paulo - UNIFESP - São Paulo (SP), Brasil.

(1) Pós-graduanda (Mestrado) em Distúrbios da Comunicação Humana pela Universidade Federal de São Paulo - UNIFESP - São Paulo (SP), Brasil.

(2) Fonoaudióloga clínica com especialização em Linguagem pela Universidade de São Paulo - USP - São Paulo (SP), Brasil.

(3) Doutora, Professora Colaboradora do Departamento de Fonoaudiologia da Universidade Federal de São Paulo - UNIFESP - São Paulo (SP), Brasil.

(4) Doutora, Professora Associada do Departamento de Fonoaudiologia da Universidade Federal de São Paulo - UNIFESP - São Paulo (SP), Brasil.

(5) Doutora, Professora Associada do Departamento de Fonoaudiologia da Universidade Federal de São Paulo - UNIFESP - São Paulo (SP), Brasil.

(6) Professora Titular do Curso de Fonoaudiologia da Universidade Federal de São Paulo - UNIFESP - São Paulo (SP), Brasil.

Endereço para correspondência: Elaine Colombo Sousa. R. Manuel Quirino de Mattos, 1927, Jardim Sapopemba, São Paulo (SP), Brasil, CEP: 03969-000.

E-mail: elainefonop@yahoo.com.br

Recebido em: 24/11/2008; Aceito em: 17/2/2009

\section{INTRODUÇÃO}

Nos quadros que compõem os transtornos do espectro autístico, tais como o autismo infantil, síndrome de Asperger, e o transtorno global do desenvolvimento sem outra especificação, existe um déficit severo de comunicação e interação social, além de padrões de comportamento estereotipados ${ }^{(1)}$.

Comumente, dentre as diversas manifestações clínicas, as atipias de respostas aos estímulos verbais e não verbais têm sido o primeiro sinal a chamar a atenção dos pais. A falta de participação em conversações pré-linguísticas associada às reações assistemáticas aos sons sugere a hipótese inicial de prejuízo na audição $0^{(2)}$.

Em estudo pioneiro verificou-se que $80 \%$ dos casos diagnosticados posteriormente, como Autismo Infantil, apresentavam como suspeita diagnóstica inicial a deficiência auditiva ${ }^{(3)}$. 
Neste sentido, a compreensão médica sobre a evolução do desenvolvimento da criança diagnosticada como pertencente ao espectro autístico é fundamental, visto que o diagnóstico tardio tem implicações cruciais na remediação dos desvios observados $^{(4-6)}$.

Levantou-se como hipótese para este estudo que a ausência de comunicação verbal pode levar a maior suspeita de perda auditiva em indivíduos com Transtornos do Espectro Autístico, embora haja outras manifestações clínicas específicas e evidentes.

Sendo assim, o objetivo desta pesquisa foi averiguar a associação entre a suspeita inicial de deficiência auditiva e a ausência de comunicação verbal em indivíduos com transtornos do espectro autístico.

Além disso, buscou-se confirmar a existência ou não da perda auditiva nestes indivíduos.

\section{MÉTODOS}

Este estudo foi realizado após aprovação pelo Comitê de Ética em Pesquisa da Universidade Federal de São Paulo UNIFESP (CEP 0542/06).

Os pais ou responsáveis pelas crianças foram informados sobre os procedimentos metodológicos e assinaram o Termo de Consentimento Livre e Esclarecido antes da realização de qualquer procedimento.

Trata-se de um estudo retrospectivo de 54 prontuários referentes à pacientes na faixa etária entre dois anos e onze meses e dezoito anos e seis meses (mediana de cinco anos de idade), de ambos os sexos, sendo $87 \%$ meninos e $13 \%$ meninas, de nível sócio-econômico baixo, avaliados e diagnosticados segundo os critérios diagnósticos do DSM IV $\operatorname{Tr}^{(7)}$, por equipe multidisciplinar, no Núcleo de Investigação Fonoaudiológica de Linguagem e Fala nos Transtornos Globais do Desenvolvimento do Departamento de Fonoaudiologia da UNIFESP, no período de março de 2000 a março de 2006.

A amostra foi composta por 39 autistas (72\%), 12 indivíduos com síndrome de Asperger (22\%), dois com síndrome de Rett (4\%) e um com transtorno global do desenvolvimento sem outra especificação (1\%).

Quanto ao comportamento comunicativo 55,56\% dos indivíduos foram considerados não verbais e 44,44\% verbais, de acordo com os critérios propostos por Fernandes ${ }^{(6)}$ que define como não verbal, a criança que produz apenas vocalizações como meio de comunicação predominante e verbal aquela que produz palavras constituídas, de pelo menos, $75 \%$ dos fonemas da Língua Portuguesa.
Inicialmente, verificamos a presença de suspeita inicial de perda auditiva como primeira etiologia referida pelo informante na anamnese. Em seguida, averiguamos o grau de associação entre a presença de suspeita inicial de perda auditiva e o tipo de comportamento comunicativo. Por fim, verificamos a existência ou não de perda auditiva, por meio do levantamento dos resultados das avaliações audiológicas dos indivíduos com e sem queixa auditiva durante o período da avaliação fonoaudiológica.

Dos exames audiológicos efetuados para a averiguação da audição após avaliação otorrinolaringológica, 50\% foram constituídos por potencial evocado auditivo de tronco encefálico e $50 \%$ por audiometria tonal liminar e imitanciometria. Importante salientar que as avaliações realizadas por meio de audiometria tonal liminar e imitanciometria foram dos indivíduos com melhor desempenho verbal.

Para a análise dos dados, empregamos o teste de Quiquadrado, complementado pela estatística de Fisher. Estabelecemos para este trabalho um nível de significância de 0,05 (5\%) e $95 \%$ de confiança estatística.

\section{RESULTADOS}

Averiguamos que $44,44 \%$ dos indivíduos da amostra apresentaram comportamento comunicativo verbal e 55,56\% apresentaram comportamento comunicativo não verbal durante o processo de avaliação fonoaudiológica.

Constatamos presença estatisticamente significante $(\mathrm{p}=0,020)$ de suspeita inicial de perda auditiva como primeira etiologia, relatada pelos pais de crianças autistas (verbais ou não), visto que $62,96 \%$ dos pais apresentaram tal suspeita enquanto apenas $37,04 \%$ dos familiares não a mencionaram.

$\mathrm{Na}$ análise do grau de associação entre a presença de suspeita inicial de perda auditiva e o tipo de comportamento comunicativo (Tabela 1), verificamos diferença estatisticamente significante entre os grupos $(\mathrm{p}=0,020)$ sendo que, a suspeita inicial foi relatada mais frequentemente no grupo de indivíduos com comportamento comunicativo não verbal $(42,59 \%)$ do que no grupo de indivíduos com comportamento comunicativo verbal $(20,37 \%)$.

Ao apurar os resultados obtidos nas avaliações audiológicas, concluímos que houve frequência estatisticamente significante maior $(\mathrm{p}=0,020)$ de diagnóstico audiológico normal $(88,89 \%)$ (Tabela 2), tanto no grupo de indivíduos com comportamento comunicativo não verbal, quanto no grupo verbal.

Verificamos maior incidência de deficiência auditiva em indivíduos não verbais $(11,11 \%)$ do que em verbais ( $0 \%)$. Além

Tabela 1. Distribuição e análise do grau de associação entre as variáveis suspeita de perda auditiva e comportamento comunicativo

\begin{tabular}{|c|c|c|c|c|c|c|}
\hline \multirow{3}{*}{$\begin{array}{l}\text { Suspeita de } \\
\text { perda auditiva }\end{array}$} & \multicolumn{4}{|c|}{ Comportamento comunicativo } & \multirow{2}{*}{\multicolumn{2}{|c|}{ Total }} \\
\hline & \multicolumn{2}{|c|}{ Verbal } & \multicolumn{2}{|c|}{ Não verbal } & & \\
\hline & Frequência & $\%$ & Frequência & $\%$ & Frequência & $\%$ \\
\hline Sim & 11 & 20,37 & 23 & $42,59^{*}$ & 34 & 62,96 \\
\hline Não & 13 & 24,07 & 7 & 12,96 & 20 & 37,04 \\
\hline Total & 24 & 44,44 & 30 & 55,56 & 54 & 100 \\
\hline
\end{tabular}

Teste de Qui-quadrado: $p=0,020 ;$ * Valor estatisticamente significante 
Tabela 2. Distribuição e análise do grau de associação entre as variáveis diagnóstico auditivo e comportamento comunicativo

\begin{tabular}{|c|c|c|c|c|c|c|}
\hline \multirow{3}{*}{$\begin{array}{l}\text { Comportamento } \\
\text { comunicativo }\end{array}$} & \multicolumn{4}{|c|}{ Diagnóstico auditivo } & \multirow{2}{*}{\multicolumn{2}{|c|}{ Total }} \\
\hline & \multicolumn{2}{|c|}{ Alterado } & \multicolumn{2}{|c|}{ Normal } & & \\
\hline & Frequência & $\%$ & Frequência & $\%$ & Frequência & $\%$ \\
\hline Não verbal & 6 & 11,11 & 24 & $44,44^{*}$ & 30 & 55,56 \\
\hline Verbal & 0 & 0,00 & 24 & $44,44^{*}$ & 24 & 44,44 \\
\hline Total & 6 & 11,11 & 48 & $88,89^{*}$ & 54 & 100 \\
\hline
\end{tabular}

Teste de Qui-quadrado, ajustado pela Estatística de Fisher: $p=0,020$; * Valores estatisticamente significantes

Tabela 3. Correlação entre suspeita inicial de perda auditiva, comportamento comunicativo e diagnóstico auditivo

\begin{tabular}{|c|c|c|c|c|c|c|c|}
\hline \multirow{3}{*}{$\begin{array}{l}\text { Comportamento } \\
\text { comunicativo }\end{array}$} & \multirow{3}{*}{$\begin{array}{c}\text { Suspeita de } \\
\text { perda auditiva }\end{array}$} & \multicolumn{4}{|c|}{ Diagnóstico auditivo } & \multirow{2}{*}{\multicolumn{2}{|c|}{ Total }} \\
\hline & & \multicolumn{2}{|c|}{ Alterado } & \multicolumn{2}{|c|}{ Normal } & & \\
\hline & & Frequência & $\%$ & Frequência & $\%$ & Frequência & $\%$ \\
\hline \multirow[t]{2}{*}{ Não verbal } & Sem suspeita & 2 & 3,70 & 5 & 9,26 & 7 & 12,96 \\
\hline & Com suspeita & 4 & 7,41 & 19 & 35,19 & 23 & 42,59 \\
\hline \multirow[t]{2}{*}{ Verbal } & Sem suspeita & 0 & 0,00 & 13 & 24,07 & 13 & 24,07 \\
\hline & Com suspeita & 0 & 0,00 & 11 & 20,37 & 11 & 20,37 \\
\hline Total & & 6 & 11,11 & 48 & 88,89 & 54 & 100 \\
\hline
\end{tabular}

disso, indivíduos não verbais com suspeita de perda auditiva apresentaram maior porcentagem de diagnóstico auditivo alterado $(7,41 \%)$ do que indivíduos não verbais sem suspeita $(3,70 \%)$ (Tabela 3).

Dos seis exames que apontaram diagnóstico audiológico alterado, cinco indicaram perdas condutivas de grau leve. Apenas um caso indicou perda auditiva neurossensorial de grau moderadamente severo, bilateralmente. O indivíduo com deficiência auditiva neurossensorial foi diagnosticado com autismo infantil e, dos sujeitos que apresentavam deficiências condutivas, quatro foram diagnosticados como autistas e um com síndrome de Rett.

\section{DISCUSSÃO}

A variação no comportamento comunicativo obtida neste estudo confirma as descrições que referiram que indivíduos com diagnóstico de Transtornos do Espectro Autístico podem ter um prejuízo da comunicação, que varia desde a ausência total de fala até a uma inabilidade no uso da linguagem com função comunicativa ${ }^{(8)}$.

A frequência de suspeita inicial de perda auditiva relatada pelos pais das crianças autistas (verbais ou não) foi inferior à obtida no estudo pioneiro que constatou $80 \%$ de suspeita inicial de deficiência auditiva em casos diagnosticados, posteriormente, como autismo infantil ${ }^{(3)}$.

A maior frequência de relato de suspeita inicial de perda auditiva no grupo de indivíduos com comportamento comunicativo não verbal corrobora o achado de estudo prévio, que também averiguou maior frequência de suspeita de perda auditiva em crianças autistas não verbais do que nas verbais ${ }^{(9)}$. Assim, constatamos que a alteração severa da linguagem, como ausência de oralidade, pode levar a maior suspeita de perda auditiva em crianças autistas ${ }^{(10)}$.

Verificamos maior ocorrência de perda auditiva em indivíduos não verbais do que em indivíduos verbais. Este dado coincide com um estudo que afirmou que os problemas auditivos podem estar positivamente relacionados à severidade dos sintomas autísticos, ou seja, prejuízos auditivos podem acentuar as dificuldades relacionais e comunicativas de indivíduos pertencentes ao espectro autístico ${ }^{(11)}$.

Embora a ocorrência de perda auditiva encontrada neste estudo seja significativa, ou seja, 1:54, sendo que na população em geral ela é de 1-6:1000 ${ }^{(12)}$, ao rastrearmos os dados de anamnese do caso com prejuízo audiológico permanente, pudemos identificar riscos para o desenvolvimento auditivo, como intercorrências peri- natais.

A maior ocorrência de diagnóstico auditivo alterado no grupo de indivíduos não verbais com suspeita de perda auditiva, em comparação ao grupo de indivíduos não verbais sem suspeita, mostra que o detalhamento das informações relatadas pelas famílias sobre o desenvolvimento da criança é fundamental para a precisão do diagnóstico e das condutas multidisciplinares.

O mesmo tipo de correlação não pôde ser realizado entre os indivíduos verbais com e sem suspeita de perda auditiva, pois estes não apresentaram comprometimento auditivo.

A porcentagem de suspeita de perda auditiva relatada pelos pais, confrontada à porcentagem de indivíduos com prejuízo auditivo confirmado, demonstra que as manifestações comportamentais de indivíduos com diagnóstico de Transtornos do Espectro Autístico em relação aos sons podem estar mais associadas às dificuldades no processamento da informação e nem tanto à acuidade auditiva ${ }^{(13)}$.

Deste modo, os indivíduos autistas podem ter dificuldades na extração de significado da informação social. Se estímulos não são significativos, eles podem não desenvolver motivação para manter atenção a este tipo de informação ${ }^{(14-16)}$.

Devemos salientar, a partir dos resultados encontrados neste estudo, a importância do conhecimento dos profissionais da área de saúde sobre as manifestações clínicas encontradas nos quadros que compõem o espectro autístico, pois a suspeita inicial de perda auditiva referida pelo informante no momento da anamnese, induz o clínico ao entendimento da alteração de linguagem como consequência de privação auditiva. Desta forma, o diagnóstico diferencial pode ser atrasado assim como a intervenção terapêutica adequada.

A suspeita de alteração auditiva pode retardar o diagnóstico dos Transtornos do Espectro Autístico. Diversos estudo têm 
apontado para certa dificuldade dos pediatras em diferenciar os sintomas do espectro autístico, de outras patologias ${ }^{(3-4)}$. A ausência da observação direta ou do relato dos comportamentos de comunicação pode induzir o clínico a considerar apenas a ausência de fala e conduzem-no ao raciocínio explicativo de caracterização da audição como diagnóstico diferencial ${ }^{(3)}$.

É importante ressaltar que o conhecimento clínico sobre as múltiplas formas pelas quais o espectro autístico pode se manifestar é fundamental, pois o diagnóstico tardio tem implicações cruciais na remediação dos prejuízos. O olhar cuidadoso da equipe multidisciplinar é imprescindível para a efetividade do diagnóstico precoce e da intervenção apropriada ${ }^{(4-5,15-16)}$.

\section{CONCLUSÃO}

O número de crianças com suspeita inicial de perda auditiva mostrou-se significativo, independente destas serem verbais ou não. Na análise da associação entre desempenho comunicativo e suspeita de prejuízos na audição os resultados foram estatisticamente significante maiores em crianças não verbais. Esses dados nos fazem refletir sobre a importância da sensibilização dos profissionais que atuam em clínica infantil quanto à busca pelo diagnóstico diferencial entre a deficiência auditiva e os transtornos do espectro autístico.

\begin{abstract}
Purpose: To determine the association between the initial suspicion of hearing loss and the absence of verbal communication in children with diagnosis of autistic spectrum disorders. Methods: A retrospective study was carried out analyzing 54 anamneses of individuals with diagnosis of autistic spectrum disorders. From each case history, it was obtained the records of the initial suspicion of hearing loss, mentioned by family members. The degree of association between the initial suspicion of hearing loss and the type of communicative performance (verbal and non-verbal) was verified. After that, hearing assessments were tracked down to confirm the pertinence of the hearing complaint mentioned. The data were statistically analysed using Chi-Square Test and Fisher's Exact Test. A significance level of 0.05 was adopted. Results: It was verified that $62.96 \%$ of the parents of children with autism (both verbal and non-verbal) exhibited an initial suspicion of hearing loss as the first etiology. The hearing assessment demonstrated that only $11.11 \%$ of the individuals with non-verbal communication and none of those with verbal communication actually had some type of hearing impairment. Conclusions: The number of children with initial suspicion of hearing loss was significant, regardless whether these individuals had verbal or non-verbal communication. However, in the association analysis between communicative performance and suspicion of hearing impairments, the results were significantly greater for non-verbal children. This fact leads to a reflection on the importance of sensitization of the professionals who act in childcare clinic regarding the search for a differential diagnosis between hearing impairment and autistic spectrum disorders.
\end{abstract}

Keywords: Hearing loss/complications; Autistic disorders; Child language; Verbal behavior; Language disorders

\title{
REFERENCIAS
}

1. Cohen H, Rémillard S. Autism and Asperger's Syndrome: a spectrum of disability. In: Brown K, editor. Encyclopedia of Language and Linguistics. 2nd ed. Oxford: Elsevier; 2006. p. 617-21.

2. Perissinoto J. Histórico do autismo. In: Perissinoto J, organizador. Conhecimentos essenciais para atender bem a criança com autismo. São José dos Campos: Pulso Editorial; 2003. p. 15-9.

3. Schopler E, Mesibov GB, editors. High-functioning individuals with autism. New York: Plenum Press; c1992.

4. Mandell DS, Novak MM, Zubritsky CD. Factors associated with age of diagnosis among children with autism spectrum disorders. Pediatrics. 2005;116(6):1480-6.

5. Turner LM, Stone WL, Pozdol SL, Coonrod EE. Follow-up of children with autism spectrum disorders from age 2 to age 9. Autism. 2006;10(3):243-65.

6. Fernandes FDM. Autismo Infantil: repensando o enfoque fonoaudiológico - aspectos funcionais da comunicação. São Paulo: Lovise; 1996.

7. American Psychiatric Association. DSM-IV-TR: Manual diagnóstico e estatístico de transtornos mentais. Porto Alegre: Artes Médicas; 2002.

8. Verté S, Geurts HM, Roeyers H, Rosseel Y, Oosterlaan J, Sergeant JA. Can the Children's Communication Checklist differentiate autism spectrum subtypes? Autism. 2006;10(3):266-87.

9. Tamanaha AC, Perissinoto J, Pedromônico MR. A perda auditiva como primeira etiologia para portadores de autismo infantil. In: $9^{\circ}$ Congresso Brasileiro de Fonoaudiologia, 2001. Belo Horizonte. Anais. Belo Horizonte: Sociedade Brasileira de Fonoaudiologia; 2001.

10. Rogel-Ortiz FJ. Autismo. Gac Méd Méx. 2005;141(2):143-7.

11. Tas A, Yagiz R, Tas M, Esme M, Uzun C, Karasalihoglu AR. Evaluation of hearing in children with autism by using TEOAE and ABR. Autism. 2007;11(1):73-9.

12. Olusanya BO, Newton VE. Global burden of childhood hearing impairment and disease control priorities for developing countries. Lancet. 2007;369(9569):1314-7. Erratum in: Lancet. 2007;369(9576): 1860.

13. Dunn MA, Gomes H, Gravel J. Mismatch negativity in children with autism and typical development. J Autism Dev Disord. 2008;38(1):5271.

14. Paul R. Auditory processing disorder. J Autism Dev Disord. 2008;38(1):208-9.

15. Tamanaha AC, Perissinoto J, Chiari BM. Uma breve revisão histórica sobre a construção dos conceitos do Autismo Infantil e da Síndrome de Asperger: [revisão]. Rev Soc Bras Fonoaudiol. 2008;13(3):296-9.

16. Tamanaha AC, Perissinoto J, Chiari BM. Evolução da criança autista a partir da resposta materna ao Autism Behavior Checklist. Pro-Fono. 2008;20(3):165-70. 